九州大学学術情報リポジトリ

Kyushu University Institutional Repository

\title{
A moving model of a CG head and its parametric expression of gender and age
}

\section{高木，英行}

Department of Acoustic Design, Kyushu Institute of Design

TAKEDA, Takashi

Department of Mechanical Engineering, Nagasaki Institute of Applied Science

http://hdl. handle. net/2324/4486275

出版情報: IEEE SMC'99 conference proceedings : 1999 IEEE International Conference on Systems, Man, and Cybernetics. 4, pp. 218-223, 1999-10-12. IEEE

バージョン :

権利関係 : 


\title{
A Moving Model of a CG Head and Its Parametric Expression of Gender and Age
}

\author{
Hideyuki TAKAGI* Takashi TAKEDA** ChinHuat EWE** \\ * Dept. of Art and Information Design, Kyushu Institute of Design \\ Shiobaru, Minami-ku, Fukuoka 815-8540, Japan, E-mail: takagiökyushu-id.ac.jp \\ ** Dept. of Mechanical Engineering, Nagasaki Institute of Applied Science \\ 536, Aba, Nagasaki, 851-0193 Japan, E-mail: takeda (âcsce.nias.ac.jp
}

\begin{abstract}
We evaluate the extent to which gender and age can be expressed in the movement of a CG human head through subjective tests. We first introduce a model of CG head movement. Then, we change the model parameters and apply it to the CG heads of a child, an asexual robot, an adult male, an adult female, and an older male. From the statistical analyses of the subjective test results of the various head movement trajectories, we found that (1) we do not need to attend to the movement differences of gender and age but just the use of the face of each gender and age, and (2) there are possible movement parameter values that emphasize and de-emphasize the expression of gender and age, though they must be weak even if they surely exist.
\end{abstract}

\section{INTRODUCTION}

The need to naturally control the movement of a CG human has increased with the rising demand for the technology in the agent communication, $\mathrm{CG}$ animation, and computer entertainment fields. Natural facial expression and movement is especially important in body communication. This is seen in the increase in research on human faceology [1]. We proposed a model of a head and line of sight movement and evaluated its realism as part of our faceological research [7].

In this paper, we thoroughly evaluate the natural likeness of a male, a female, a child, and an older male using the proposed model. If head movement can express a difference in gender and age, we must determine its influence on, importance to, and usefulness in CG creation. Even if there is no such relationship between head movement and gender and age, the information can be used to create realistic facial expressions and head movement of a male, a female, and a young and old person.
Males and females differ in their body motion due to their anatomical differences. Their daily unconscious behavior is also different [5, 3]. However, it is unknown whether there is such a gender difference in the motion of the human head.

The objective of this paper is to clarify whether there are factors affecting the motion of a head and to obtain such useful information for CG creation. In our research, we propose a model, change its parameters, and analyze the relationship between the parameters and subjective test results.

\section{A Parametric Model of Head Movement}

The time trajectory characteristics of our proposed head and its line of sight model is shown in the equation $f(t)$ and Figure 1 .

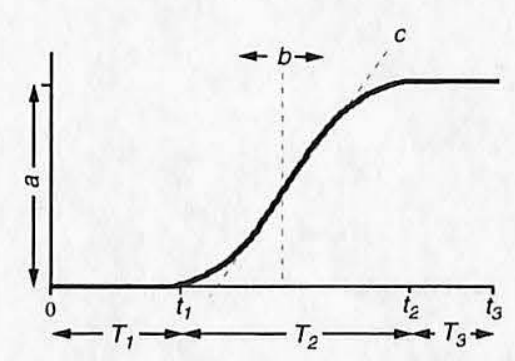

Figure 1: The role of parameters of the proposed model.

$$
\begin{aligned}
& f(t)= \begin{cases}0 & 0 \leq t \leq t_{1} \\
\frac{A}{1+b \exp \left\{-c\left(t-T_{1}-T_{2} / 2\right)\right\}}-D & t_{1} \leq t \leq t_{2} \\
a & t_{2} \leq t \leq t_{3}\end{cases} \\
& A=\frac{a}{b} \cdot \frac{\left(1+b \exp \left\{c \frac{T_{2}}{2}\right\}\right)\left(1+b \exp \left\{-c \frac{T_{2}}{2}\right\}\right)}{\exp \left\{c \frac{T_{2}}{2}\right\}-\exp \left\{-c \frac{T_{2}}{2}\right\}} \\
& D=\frac{a}{b} \cdot \frac{1+b \exp \left\{-c \frac{T_{2}}{2}\right\}}{\exp \left\{c \frac{T_{2}}{2}\right\}-\exp \left\{-c \frac{T_{2}}{2}\right\}}
\end{aligned}
$$


where $t_{i}$ and $T_{i}$ are the time and time intervals of before, during, and after the movements shown in Figure 1 (the model needs only $T_{i}$.) $a, b$, and $c$ are shape parameters of sigmoidal moving characteristics; where $a, b$, and $c$ adjust the stop target position, the symmetry before and after movement, and the change of angular velocity of movement, respectively (see Figure 1.)

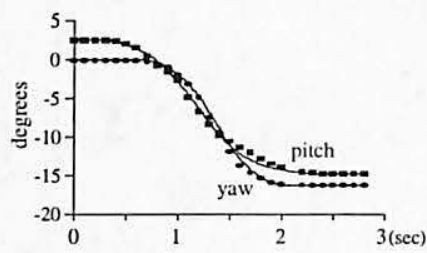

(a) $(\phi, \theta)=(-30,-60)$

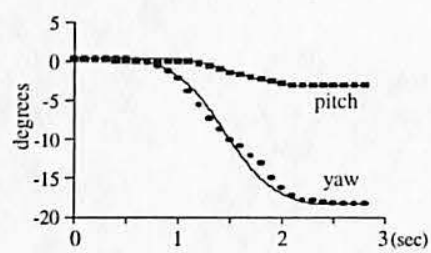

(b) $(\phi, \theta)=(-30,-30)$

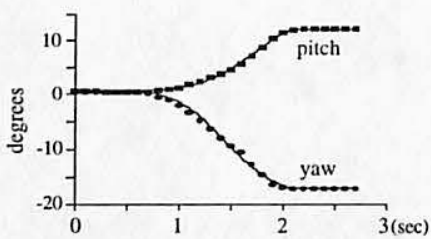

(c) $(\phi, \theta)=(-30,30)$

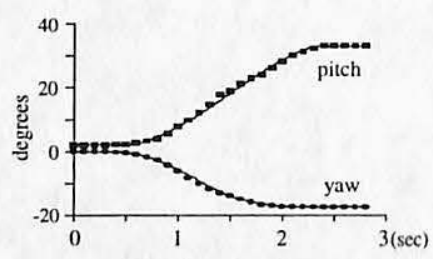

(d) $(\phi, \theta)=(-30,60)$

Figure 2: Head movement trajectory based on our proposed model mapped onto the measurement of head movement. Dotted lines represent actual measurement data, and solid lines represent the output of our proposed model. $(\phi, \theta)=$ (horizontal angle, elevation angle) is the target coordinates of the given line of sight.

For example, we set $a=40$ degrees if a head movement is stopped at the target pitch angle position of 40 degrees; we set $b=1$ if the moving characteristics in the first half and the latter half of the movement are the same, set $b<1$ if the movement starts slowly then quickly reaches the target, and set $b>1$ for the opposite characteristic of movement; and we set $c$ to a small value for a linear-like movement of angular change and to a large value for the opposite.

This moving model is based on the fact that the measured head trajectory is sigmoidal. This measurement is physically natural because the mass of human head is so big that its angular acceleration at start and stop phases becomes large. Figure 2 shows how well our model fits actual head movements.

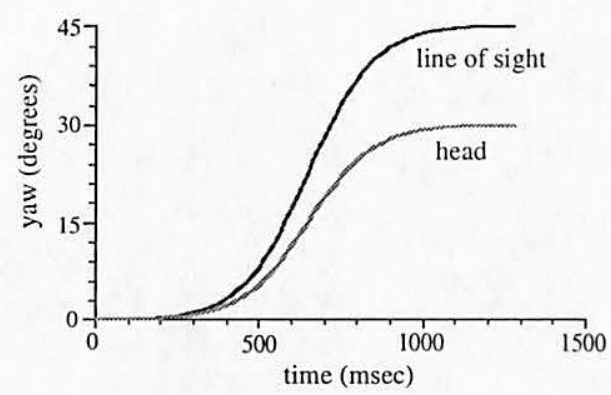

(a)

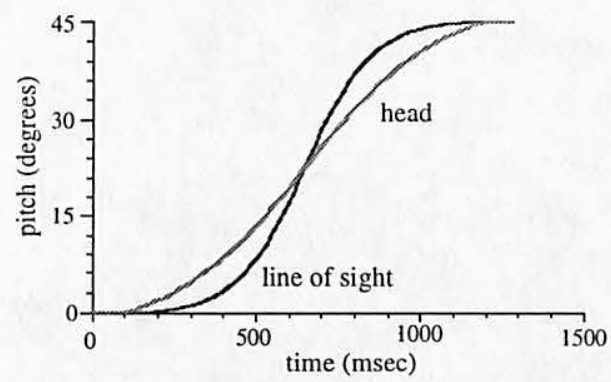

(b)

Figure 3: Two examples of movement based on the proposed model: (a) the case of talking with two people in front of the CG agent, and (b) the case of looking up from an article on a desk toward a visitor.

The combination of this model for a head and eyes resemble several expressions which are natural, comical, strange, and so on. Figure 3 (a) is an example of a movement when a CG agent talks with two people in front of it. The eyes move first, and then the head starts to move. When the CG agent's line of sight directly catches the target face, the head has yet to meet the target person face to face. When the line of sight movement stops, the head does not reach the same yaw angle. If looking at the target face requires only a change in pitch, the $\mathrm{CG}$ agent looks with only its eyes turned upward.

Figure 3 (b) is the case of a CG agent looking up from an article on a desk toward a visitor's face. Initially, the head starts to move upward from an article on a desk. Soon, the agent's line of sight catches the visitor's face before the movement of its head is completed. Finally, the CG agent directly 
looks at the visitor's face. The eyes in this case move up and down.

\section{Subjective Tests for Gender and Age Expressions on CG Head Movement}

\subsection{CG faces and subjects for subjective tests}

Figure 4 shows the faces we used in the subjective tests in section 3.3. An asexual robot-like face is used to contrast with the male and the female faces.

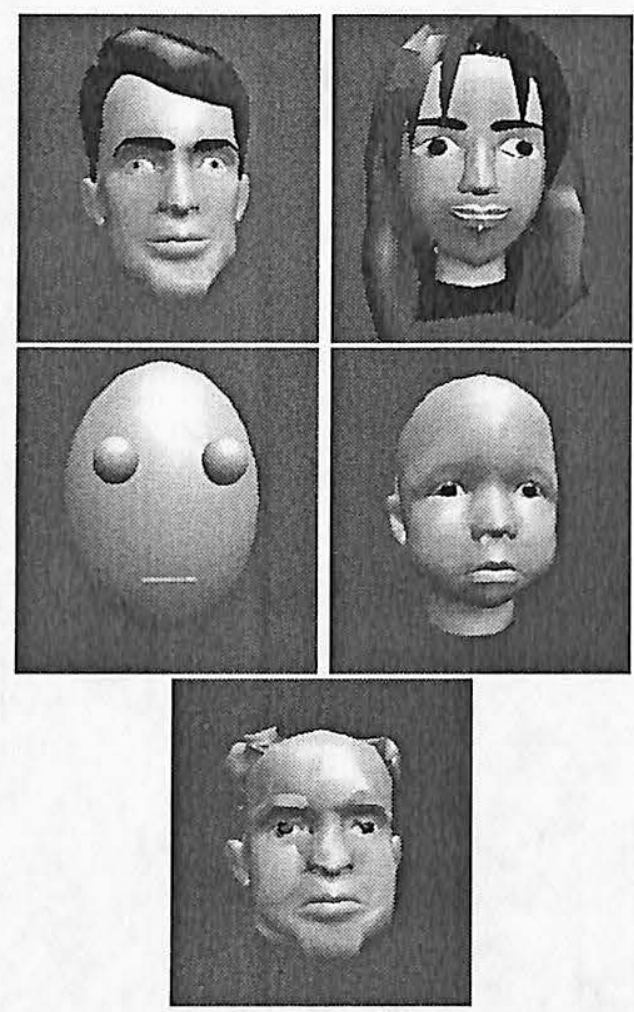

Figure 4: CG faces used for the evaluation of head movements. The asexual face is made to be compared with the male and female ones from parameter dependency on gender point of view.

Our subjects are undergraduate students in their 20 's. A group of 29 and of 24 students subjectively test the model parameters' dependence on gender expression (section 3.3.1) and the roll angle's dependence on gender and age expressions (section 3.3.2), respectively.

\subsection{Constraints in experiments}

In this paper, we define the movement of a head with yaw, pitch, and roll angles (see Figure 5.)

To keep the same experimental conditions and avoid inconsistency due to subject fatigue, we have to introduce constraints in the combination of experimental parameters and their values.

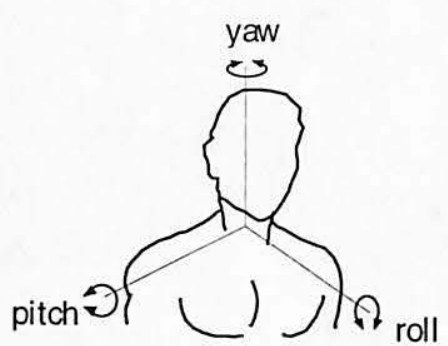

Figure 5: Angles of a head movement.

Our first constraint is not to combine the model parameters of yaw, pitch, and roll angles to avoid the third power order of combination. Model parameters for the three angles change together with the same values except parameter $a$.

Our second constraint is to fix the movement of the eyes to avoid the second power order of combination. The line of sight moves to a target coordinate, always following the center point on a front plane. Due to this constraint, the $a$ parameter of the yaw and pitch angles are uniquely determined by the horizontal and elevation angles, respectively.

\subsection{Experimental conditions}

We conduct two subjective tests. In both tests, all subjects are gathered in the same room at the same time to evaluate CG images projected on a screen.

\subsubsection{Subjective test for gender expression based on model parameters}

The values of head movement parameters listed in Table 1 are used in this subjective test.

Table 1: Parameter values used for the subjective test of gender expression. Parameters of yaw, pitch, and roll angles change together with the same values except for parameter $a$.

\begin{tabular}{|l|c|c|c|}
\hline & yaw & pitch & roll \\
\hline$a$ (degrees) & 37 & $(-15,16)$ & 0.0 \\
\hline$T_{1}$ (seconds) & \multicolumn{3}{|c|}{1.0} \\
\hline$T_{2}$ (seconds) & \multicolumn{2}{|c|}{$(1.3,1.8)$} \\
\hline$T_{3}$ (seconds) & \multicolumn{2}{|c|}{1.0} \\
\hline$b$ & $(0.1,1.0,20.0)$ \\
\hline$c$ & \multicolumn{2}{|c|}{$(3.0,9.0,15.0)$} \\
\hline
\end{tabular}

As previously mentioned in section 3.2 , the $a$ parameter of yaw and pitch angles are put the horizontal and elevation angles, respectively. The $a$ of the roll angle is always set to 0 degrees, which means the movement does not cock the head to one side. Since $T_{1}$ and $T_{3}$ are the time periods when a head is not 
moving, $T_{2}$ plays an important role in distinguishing gender among time parameters of trajectory, $T_{i}$.

In Table 1, four gender-related impression parameters are analyzed. The number of displayed movements for each CG head is 36 (=2 (\# of target coordinates) $\times 3$ (\# of $b$ values) $\times 3$ (\# of $c$ values) .) Each movement is performed once, followed by a one-second pause.

Subjects evaluated the head movements of a male, a female, and an asexual robot according to five levels between "masculine to unmasculine," "feminine to unfeminine," and "feminine to masculine."

\subsubsection{Subjective test for gender and age expressions based on the roll angle}

The parameter values of head movement listed in Table 2 are used in this subjective test.

Table 2: Parameter values used for subjective test of gender and age expression in the roll angle. Parameters of yaw, pitch, and roll angles change together with the same values except $a$.

\begin{tabular}{|l|c|c|c|}
\hline & yaw & pitch & roll \\
\hline$a$ (degrees) & 37 & 20 & $(0.0,10.0)$ \\
\hline$T_{1}$ (seconds) & \multicolumn{3}{|c|}{1.0} \\
\hline$T_{2}$ (seconds) & \multicolumn{2}{|c|}{1.8} \\
\hline$T_{3}$ (seconds) & \multicolumn{2}{|c|}{0.5} \\
\hline$b$ & \multicolumn{3}{|c|}{$(0.1,1.0,20.0)$} \\
\hline$c$ & \multicolumn{3}{|c|}{$(3.0,9.0,15.0)$} \\
\hline
\end{tabular}

In this experiment, we analyze how the roll angle of a head contributes to the expressions of gender and age by changing the roll angle to 0 and 10 degrees. A pair of the two angles is compared; parameters $b$ and $c$ are also changed as in Table 2 . The target coordinate is fixed to $(37,20)$ degrees. The number of displayed movements pairs of each CG head is 18 (= 3 (\# of $b$ values) $\times 3$ (\# of $c$ values) $\times$ 2 (to avoid an order effect).) A six-second pause is inserted between comparison pairs and a one-second pause is inserted between two movements. All the CG faces in Figure 4 except the robot face are used in this experiment.

Subjects evaluate the masculine, feminine, youth$f u l$, and elderly of each pair movement in five levels from $X X$-like to $X X$-unlike.

\section{Analysis of Subjective Tests and DISCUSSION}

\subsection{Gender expression of model parameters}

\subsubsection{Analysis of variance and HSD test}

The subjective test results in the previous section were obtained by using analysis of variance. When the significance level of $b$ and $c$ obtained by the analysis of variance is less than 0.05 , a multiple comparison test is applied to the three parameter values to make it clear which two of the three parameter values are significantly different. We use Tukey's HSD test [4] for this purpose. Table 3 shows these statistical test results.

Table 3: The results of variance analysis and HSD test for the subjective test data for movements of a $\mathrm{CG}$ head. The table shows model parameters, the average of subjective evaluation in five levels, parameters that have significant difference, and significance marks; where E.A. means the elevation angle, * and ** mean $(p<0.05)$ and $(p<0.01)$, respectively.

(a) The movements of a CG male head.

$\begin{array}{ll}\text { E.A.(-15): } & 2.96 \\ \text { E.A.(16): } & 3.07 \\ \mathrm{~T}_{2}(1.3): & 3.03 \\ \mathrm{~T}_{2}(1.8): & 2.99 \\ \text { b }(0.1): & 3.21 \\ \text { b }(1.0): & 3.01 \\ \text { b }(20.0): & 2.82 \\ \text { c }(3.0): & 2.82 \\ \text { c }(9.0): & 3.08 \\ \text { c }(15.0): & 3.14\end{array}$

(b) The movements of a female CG head.

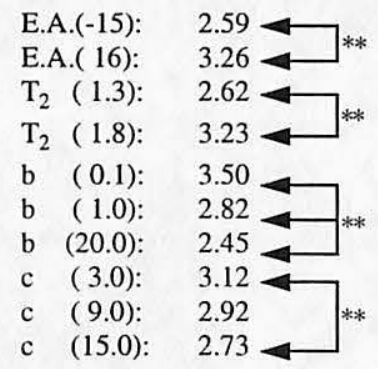

(c) The movements of an asexual robot CG head.

\begin{tabular}{|c|c|}
\hline E.A. $(-15)$ : & 2.88 \\
\hline E.A.( 16): & 3.15 \\
\hline $\mathrm{T}_{2}$ (1.3): & 2.90 \\
\hline $\mathrm{T}_{2}$ (1.8): & 3.12 \\
\hline b (0.1): & 3.12 \\
\hline b (1.0): & 2.98 \\
\hline b (20.0): & 2.94 \\
\hline c (3.0): & 3.16 \\
\hline c (9.0): & 2.94 \\
\hline c (15.0): & 2.95 \\
\hline
\end{tabular}

Overall, we can say that any parametric movements with any of the faces do not specifically work to distinguish gender; the average of subjective evaluation is in the category of fair, $3 \pm 0.5$ in five rating levels. Although there are slight differences, statistical tests have shown that the following points are significantly different. When a male head moves: 
- The movement that starts more slowly - 0.1 of parameter $b$ than 20 - looks masculine.

- The movement whose angular velocity is faster - 3 of parameter $c$ than 15 - looks masculine.

When a female head moves, several parameters contribute to feminine movements:

- Raising the head (16 degrees of elevation angle) looks more feminine than looking down ( -15 degrees of elevation angle).

- Slow movement ( $T_{2}=1.8$ seconds) looks more feminine than a quick movement $\left(T_{2}=1.3 \mathrm{sec}\right.$ onds).

- Movement that starts slowly - smaller values of parameter $b$ - looks feminine.

- Smaller changes of angular velocity of movement - 3 of parameter $c$ than $15-$ looks feminine.

When an asexual robot head moves:

- Slow movement $\left(T_{2}=1.8\right.$ seconds) looks more feminine than a quick movement $\left(T_{2}=1.3 \mathrm{sec}\right.$ onds).

\subsubsection{Method of successive categories}

We applied the method of successive categories [2] to the rating results of five levels: masculine, a little masculine, fair, a little unmasculine, and unmasculine, for example. Our analysis has shown that almost all combinations of parameters belong to the fair category. This is a matter of course from the average values in Table 3 point of view.

There was no typical evaluation - masculine, unmasculine, feminine, and unfeminine, 1st and 5th rating categories in five levels - among all head movements of male, female, and robot faces. This means that the summarized result of the method of successive categories did not belong to the typical categories, although some subjects chose a typical rating, a 1 st or 5 th rating.

There were four notable movements among those that belong to the categories of a little masculine/feminine/unfeminine, 2nd and 4th rating categories in five levels.

1. (pitch angle $\left.a, T_{2}, b, c\right)=(-15,1.3,1.0,3)$ may be a feminine movement. This movement of male, female, and robot heads was categorized in a little unmasculine, a little feminine, and fair, respectively.

2. (pitch angle $\left.a, T_{2}, b, c\right)=(-15,1.8,0.1,9)$ may be a feminine movement. This movement of male, female, and robot heads was categorized in a little unmasculine, a little feminine, and fair, respectively.
3. (pitch angle $\left.a, T_{2}, b, c\right)=(16,1.3,0.1,9)$ may be a movement that emphasizes the gender feature. This movement of male, female, and robot heads was categorized in a little masculine, a little feminine, and fair, respectively.

4. (pitch angle $\left.a, T_{2}, b, c\right)=(16,1.8,20,9)$ may be a movement that weakens the gender feature. This movement of male, female, and robot heads was categorized in a little unmasculine, a little unfeminine, and fair, respectively.

\subsection{Gender and age expression by rolling the head}

We examined the effect of the roll angle in head movements by applying the sign test [6] to the subjective test results. Table 4 shows the results.

Commonly speaking, $(b, c$, roll angle $)=(1.0,9.0$, $0.0)$ significantly looks $X X$-like than $(1.0,9.0,10.0)$ for any faces of a male, a female, a child, and an older male (significant level: $p<0.01$.) The parameter $b=1.0$ is the case that angular velocity in the former half and latter half is balanced. In such a case, head movements without rolling a head might look more natural than that with rolling.

\section{Conclusion}

We evaluated the movement of a CG head from the points of view of gender and age. Through subjective tests and statistical tests for the faces of a male, a female, a child, and an older male, we found that, basically, the movement of the head alone does not express specified features of gender and age. We have also found that the combination of some moving parameters may show their weak features. These obtained facts, especially the former one, are useful when creating CG animation and CG agents for communication.

\section{Acknowledgments}

This research was supported in part by the Research Fund of Kyushu Institute of Design.

\section{REFERENCES}

[1] "Special Issues on Face," Trans. of IEICE (the Inst. of Electr., Info. and Comm. Eng.,) vol.J80A and vol.J80-D-II, no.8 (1997) (in Japanese.)

[2] Dixon, W. J. and Massey, F. J., (1951), Introduction to statistical analysis, McGraw-Hill, New York, pp.342-343.

[3] Collett , P. and Marsh, P., "Patterns of public behavior: collision avoidance on a modern crossing," Semotica, vol.12, no.4, pp.281-299 (1974). 
[4] Hochberg, Y. and Tamhane, A. C., Multiple comparison procedures, New York: Wiley (1987).

[5] Morris, Desmond, "Manwatching: a field guide to human behavior," London: Cape (1977).

[6] S. Siegel and N. J. Jr. Castellan, "Nonparametric statistics for the behavioral science," 2nd ed., McGraw-Hill Book Company (1988).

[7] Takagi, H. and Takeda, T., "Movement models of head and eyes for computer graphics," Electronics and Communications in Japan, Part 3 , vol.82, no.1, pp.41-50, Script Tecnica (1999).
Table 4: Sign test for the subject test result to examine the effect of the roll angle to express gender and age. ** and *: a movement with roll angle $a=0.0$ is significantly natural for $X X$ like: $(p<0.01$ and $0.05) ;++$ and + : that with roll angle $a=10.0$ is significantly natural for $X X$ like: $(p<0.01$ and $0.05)$.

(a) Male adult's CG head

\begin{tabular}{|rr|rrr|c|}
\hline$b$ & $c$ & \multicolumn{3}{|c|}{ roll angle } & sign test \\
& & 0.0 & even & 10.0 & \\
\hline 0.1 & 3.0 & 17 & 4 & 3 & $* *$ \\
\hline 0.1 & 9.0 & 5 & 8 & 11 & \\
\hline 0.1 & 15.0 & 7 & 13 & 4 & \\
\hline 1.0 & 3.0 & 10 & 8 & 6 & \\
\hline 1.0 & 9.0 & 19 & 2 & 3 & $* *$ \\
\hline 1.0 & 15.0 & 8 & 5 & 11 & \\
\hline 20.0 & 3.0 & 9 & 8 & 7 & \\
\hline 20.0 & 9.0 & 6 & 7 & 11 & \\
\hline 20.0 & 15.0 & 3 & 4 & 17 & ++ \\
\hline
\end{tabular}

(b) Female adult's CG head

\begin{tabular}{|rr|rrr|l|}
\hline$b$ & $c$ & \multicolumn{3}{|c|}{ roll angle } & sign test \\
& & 0.0 & even & 10.0 & \\
\hline 0.1 & 3.0 & 9 & 7 & 8 & \\
\hline 0.1 & 9.0 & 7 & 10 & 7 & \\
\hline 0.1 & 15.0 & 5 & 7 & 12 & \\
\hline 1.0 & 3.0 & 5 & 9 & 10 & \\
\hline 1.0 & 9.0 & 21 & 0 & 3 & $* *$ \\
\hline 1.0 & 15.0 & 10 & 9 & 5 & \\
\hline 20.0 & 3.0 & 1 & 12 & 11 & ++ \\
\hline 20.0 & 9.0 & 8 & 6 & 10 & \\
\hline 20.0 & 15.0 & 8 & 4 & 12 & \\
\hline
\end{tabular}

(c) Boy's CG head

\begin{tabular}{|rr|rrr|l|}
\hline$b$ & $c$ & \multicolumn{3}{|c|}{ roll angle } & sign test \\
& & 0.0 & even & 10.0 & \\
\hline 0.1 & 3.0 & 5 & 9 & 10 & \\
\hline 0.1 & 9.0 & 12 & 6 & 6 & \\
\hline 0.1 & 15.0 & 8 & 5 & 11 & \\
\hline 1.0 & 3.0 & 10 & 5 & 9 & \\
\hline 1.0 & 9.0 & 20 & 0 & 4 & $* *$ \\
\hline 1.0 & 15.0 & 6 & 9 & 9 & \\
\hline 20.0 & 3.0 & 9 & 10 & 5 & \\
\hline 20.0 & 9.0 & 7 & 11 & 6 & \\
\hline 20.0 & 15.0 & 13 & 2 & 9 & \\
\hline
\end{tabular}

(d) Older male's head

\begin{tabular}{|rr|rrr|l|}
\hline$b$ & $c$ & \multicolumn{3}{|c|}{ roll angle } & sign test \\
& & 0.0 & even & 10.0 & \\
\hline 0.1 & 3.0 & 11 & 6 & 7 & \\
\hline 0.1 & 9.0 & 11 & 4 & 9 & \\
\hline 0.1 & 15.0 & 5 & 9 & 10 & \\
\hline 1.0 & 3.0 & 10 & 6 & 8 & \\
\hline 1.0 & 9.0 & 20 & 2 & 2 & ** \\
\hline 1.0 & 15.0 & 10 & 7 & 7 & \\
\hline 20.0 & 3.0 & 8 & 10 & 7 & \\
\hline 20.0 & 9.0 & 6 & 11 & 7 & \\
\hline 20.0 & 15.0 & 14 & 5 & 5 & \\
\hline
\end{tabular}

